\title{
Does anesthetic technique influence functional recovery after total knee arthroplasty?
}

André Carrão', Daniel Ribeiro', Patrícia O'Neill', Filipa Duarte ${ }^{1}$, Miguel Ghira ${ }^{1}$ ${ }^{1}$ Anesthesiology Department, Hospital Beatriz Ângelo, Portugal

\section{Introduction}

Faster functional recovery after surgery is the purpose of enhanced recovery in orthopedics ${ }^{1}$ and the anesthetic technique may be of influence ${ }^{2}$. This study aims to review the patients who underwent a total knee arthroplasty during two different time periods in our hospital.

\section{Methods}

We performed a retrospective analysis of the electronic health record of the patients who had a total knee arthroplasty in 2013/2014 (group A) and from September 2017 to April 2018 (group B). The statistical analysis was done with SPSS (version 23).

\section{Results}

We studied 119 patients, 61 from group A and 58 from group B. Patient characteristics were similar in the two groups (Table 1). Both intraoperative anesthesia technique and postoperative regional analgesia were significantly different between the two groups. Patients in group A had mostly a combined spinalepidural anesthesia or a subarachnoid combined with single-shot femoral block while those in group B received a subarachnoid block and a continuous femoral block (Table 2). Regarding functional recovery, patients in group B were capable of a higher knee flexion angle in the immediate postoperative period and this finding reached statistical significance in postoperative days 2 and 3 (p-value 0.004 and 0.040, respectively), as shown in Graph 1 . Time to discharge was not statistically different.

\begin{tabular}{|c|c|c|c|}
\hline & Group A & Group B & $p$-value \\
\hline \multicolumn{4}{|l|}{ Gender - n (\%) } \\
\hline Female & $50(82 \%)$ & $43(74,1 \%)$ & \multirow{2}{*}{0.302} \\
\hline Male & $11(18 \%)$ & $15(25,9 \%)$ & \\
\hline Age, years - mean $\pm S D$ & $70 \pm 6$ & $70 \pm 7$ & 0.722 \\
\hline Height, $m$ - mean \pm SD & $1,58 \pm 0,07$ & $1,60 \pm 0,08$ & 0.261 \\
\hline Weight, $\mathrm{kg}-$ mean $\pm \mathrm{SD}$ & $75,2 \pm 9,2$ & $74 \pm 11$ & 0.525 \\
\hline \multicolumn{4}{|l|}{ ASA physical status $-\mathrm{n}(\%)$} \\
\hline II & $43(70,5 \%)$ & $43(74,1 \%)$ & \multirow{2}{*}{0.657} \\
\hline III & $18(29,5 \%)$ & $15(25,9 \%)$ & \\
\hline \multicolumn{4}{|c|}{ Knee angle deformity ${ }^{*}-\mathrm{n}(\%)$} \\
\hline Genum varum & $46(83,6 \%)$ & $31(79,5 \%)$ & \multirow{2}{*}{0.607} \\
\hline Genum valgum & $9(16,4 \%)$ & $8(20,5 \%)$ & \\
\hline \multicolumn{4}{|c|}{ Surgical procedure laterality- $\mathrm{n}(\%)$} \\
\hline Right knee & $36(59 \%)$ & $29(50 \%)$ & \multirow{2}{*}{0.323} \\
\hline Left knee & $25(41 \%)$ & $29(50 \%)$ & \\
\hline
\end{tabular}

\begin{tabular}{|c|c|c|c|}
\hline \multicolumn{4}{|c|}{ Table 2 - Anesthesia Technique } \\
\hline & Group A & Group B & $p$-value \\
\hline \multicolumn{4}{|l|}{ Intraoperative anesthesia $-\mathrm{n}(\%)$} \\
\hline General anesthesia & $5(8,2 \%)$ & $6(10,3 \%)$ & \multirow{3}{*}{$<0.001$} \\
\hline Subarachnoid block & $26(42,6 \%)$ & $52(89,7 \%)$ & \\
\hline Combined spinal-epidural & $30(49,2 \%)$ & $0(0 \%)$ & \\
\hline \multicolumn{4}{|c|}{ Postoperative analgesia with peripheral nerve block - n (\%) } \\
\hline Single-shot femoral block & $14(23 \%)$ & $1(1,7 \%)$ & \multirow{3}{*}{$<0.001$} \\
\hline Continuous femoral block & $13(21,3 \%)$ & $57(98,3 \%)$ & \\
\hline No block & $34(55,7 \%)$ & $0(0 \%)$ & \\
\hline
\end{tabular}

\section{Conclusion}

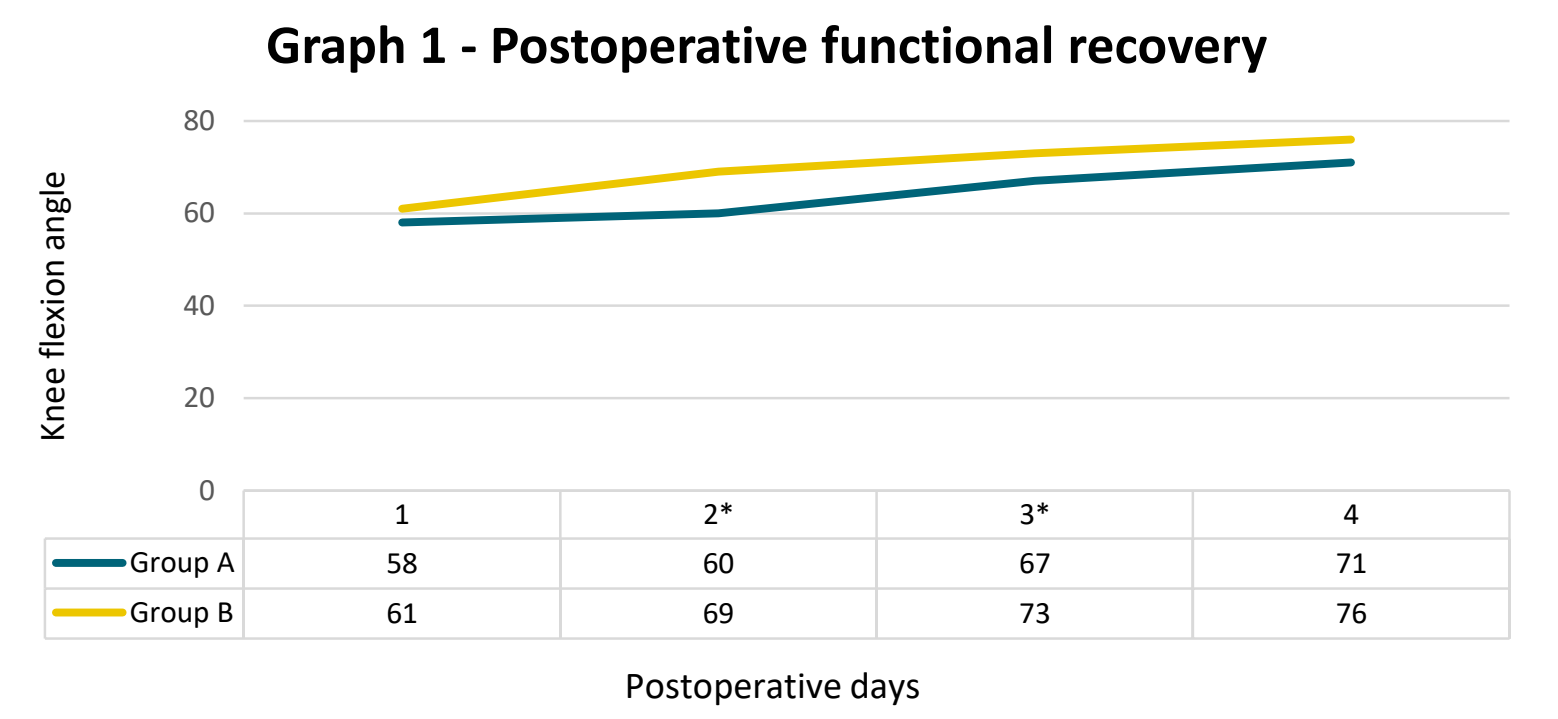

Anesthetic technique for total knee arthroplasty has changed in our hospital, from a combined spinalepidural anesthesia to a subarachnoid block followed by a continuous femoral block. Functional recovery, as shown by active knee flexion angle, has improved and this may be the result of a different anesthetic approach.

\section{References}

1. Soffin E. M., YaDeau J. T.; Enhanced recovery after surgery for primary hip and knee arthroplasty: a review of evidence; BJA; 2016; 117: 62-72

2. Kopp S. L. et al.; Anesthesia and analgesia practice pathway options for total knee arthroplasty; Reg Anesth Pain Med; 2017; 42: 683-697 